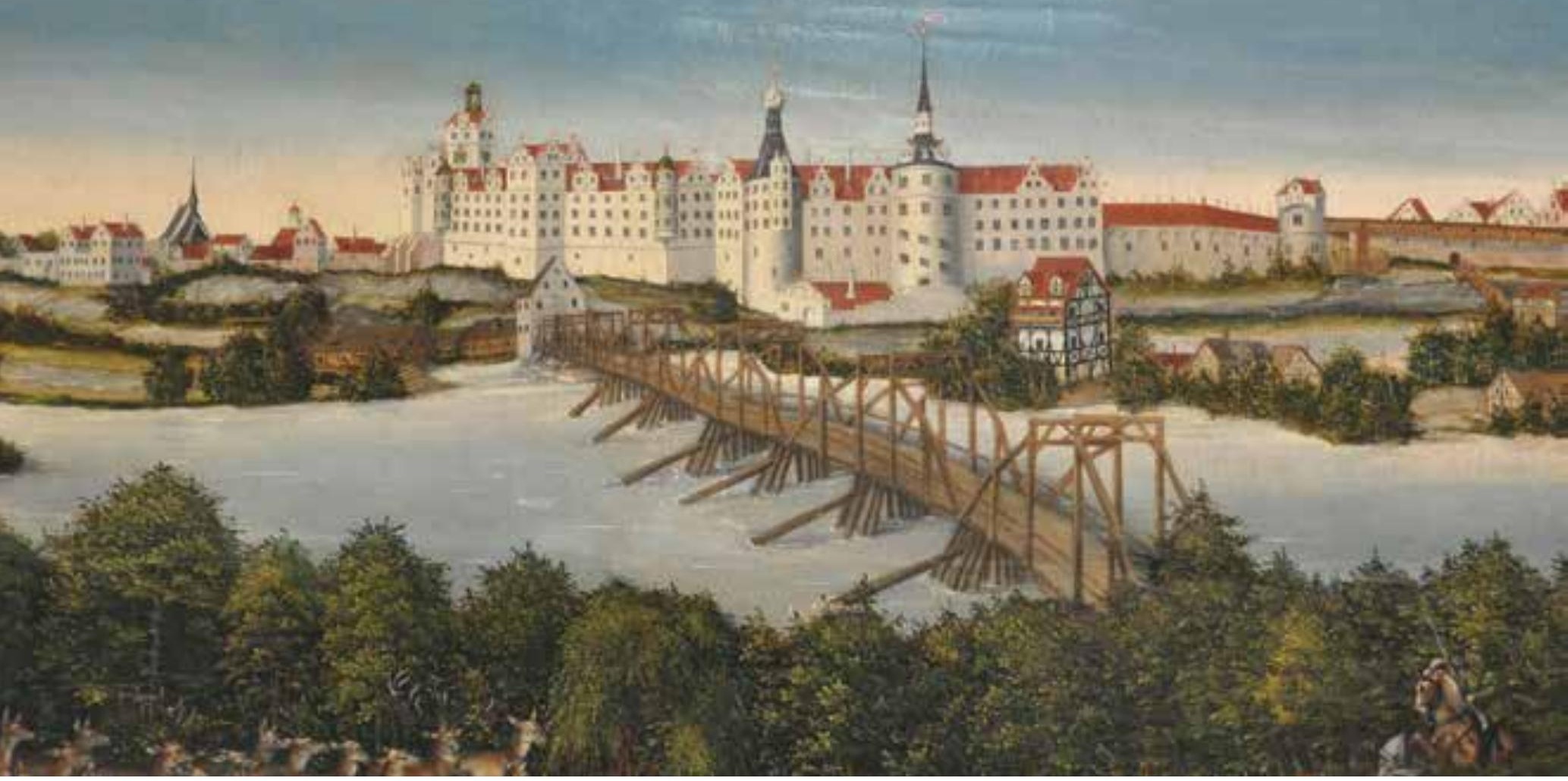

\title{
Torgau als politisches Zentrum der lutherischen Reformation
}

\author{
Hansjochen Hancke
}

Torgau vor der Mitte des

16. Jahrhunderts.

Ausschnitt aus einem Gemälde

von Lucas Cranach, 1544

๑ Kunsthistorisches Museum Wien,

GG 856
Bis zum Zweiten Weltkrieg hatte der Name Torgau Klang: durch die Rolle im Sachsen der Reformationszeit, durch die Schöpfungen auf dem Gebiete der Baukunst und der evangelischen Kirchenmusik. Zum letzten großen Auszug der Geharnischten 1938 kamen 10.000 Gäste in die Stadt, die amerikanische Botschaft in Berlin reiste mit 240 Personen in einem Sonderzug an. Das änderte sich nach dem Kriege hinsichtlich der Wertung der Reformation zugunsten der Begegnung der amerikanischen und sowjetischen Streitkräfte. Die Altstadt Torgaus wurde 1979 Flächendenkmal und ist heute städtebauliches Denkmal internationalen Ranges. Inzwischen führt die Stadt Torgau an der Elbe den Hinweis: Stadt der Renaissance und Reformation.

Wittenberg war das geistige Zentrum, Torgau das politische Zentrum der lutherischen Reformation. Torgau war kursächsische Hauptresidenz vor Dresden in den entscheidenden Jahrzehnten des 16. Jahrhunderts. Die Verbindung zwischen Wittenberg und Torgau war eng in dieser Zeit, und Philipp Melanchthon hat dieses sogar gefeiert, indem er in einem Hymnus die beiden Orte als Schwestern auftreten ließ. Torgau ist die einzige vollständig erhaltene kursächsische Residenzstadt des 16. Jahrhunderts und gilt als die wohl am besten überkommene Renaissancestadt in Deutschland, so der frühere Präsident des Landesamtes für Denkmalpflege Sachsen, Gerhard Glaser.
Gegen Ende des Mittelalters nahmen Burg und Stadt Torgau im wettinischen Herrschaftsbereich eine wichtige Stellung ein. Die Elblinie von Wittenberg über Torgau nach Meißen und Dresden war „das politisch-strategische Rückgrat der wettinischen Macht“. ${ }^{1}$ Seit dem 14. Jahrhundert hatten die Markgrafen und Kurfürsten die Burg zeitweise bewohnt und im 15. Jahrhundert zum Schloss ausgebaut. Mit dem Regierungsantritt Kurfürst Johanns des Beständigen 1525 und unter seinem Sohn und Nachfolger Johann Friedrich dem Großmütigen wurde Torgau zur wichtigsten Residenz und zur Hauptstadt des Kurfürstentums Sachsen. Starke Bautätigkeit nach dem zweiten großen Stadtbrand 1482, Blüte der Kunst unter Friedrich dem Weisen und große dynastische Festlichkeiten der Wettiner stärkten die wirtschaftliche Situation der Stadt. Es entstand eine steinerne Stadt mit auf die Stadttore ausgerichteten großzügigen Straßen, die den gleichzeitigen Einzug vieler, auch berittener Gäste ermöglichten. In der Zeit von 1505 bis 1551 verdoppelte sich die Einwohnerzahl von etwa 3.000 auf annähernd 6.000. Insgesamt war dies für die Zeit ein ungeheurer Entwicklungssprung. Cranachs Ansichten von Torgau dokumentieren diese große Blütezeit der Stadt im zweiten Viertel des 16. Jahrhunderts.

Die große Stadtansicht von Lucas Cranach dem Älteren von 1545 in Madrid ist besonders beeindruckend: Die Lage der Stadt direkt am Fluss wird deut- 
lich und auch die durch das Porphyrmassiv bedingte Entwicklung des Stadtgrundrisses in die Tiefe des Raumes hinein, weg von der Elbe. Ähnlich erlebt man auch heute den Blick vom östlichen Elbufer. Die dargestellten Kernbereiche der Stadt vom Schloss über die Kirchen zum Franziskanerkloster und der Stadtbefestigung sind noch immer charakteristisch und machen es leicht, in der Gegenwart die Stadt Cranachs wiederzufinden. Es handelt sich um Realarchitektur in nachvollziehbarer Darstellung und dieses aus einer Zeit, die nach der Natur gezeichnete Ortsansichten gerade in ihren Anfängen erlebte. Die Ansichten von Schloss und Stadt Torgau erscheinen jeweils im Hintergrund detailgetreuer Jagdszenen, offensichtlich herausragende Repräsentationsgeschenke mit politisch-diplomatischem Bezug. Eindrucksvolle Architektur wie auch prächtige Wappen zeichnen gleichermaßen den Schenker aus. Sowohl Einladung wie Erinnerungswert erfahren dadurch eine Steigerung. Gemälde mit Torgau-Ansichten befinden sich heute in Oslo, Dublin, Cleveland, Madrid und Wien. Gerade die großformatigen Jagdbilder in Madrid und Wien mit eindrucksvollen Personendarstellungen von Kaiser Karl V. und seinem Bruder König Ferdinand I. lassen sich sehr wohl in die diplomatischen Bemühungen des Kurfürsten Johann Friedrich von Sachsen um friedvollen Ausgleich mit den Habsburgern einordnen.

Die Reformation wurde in Sachsen hervorgebracht, im ernestinischen Sachsen. Den sächsischen Kurfürsten und den in ihrem Bereich wirkenden Kräften gebührt daher besondere Beachtung. Die Reformation ist der Beitrag Sachsens zur Weltgeschichte. Nach Martin Luthers Grunderkenntnis von der Gerechtigkeit Gottes verloren alle Anstrengungen ihren Wert, Gottes Gnade durch gute Werke zu verdienen: Heilsgewissheit sei allein durch den Glauben (sola fide) zu erlangen.

Kurfürst Friedrich III. von Sachsen, den man schon zu Lebzeiten den Weisen nannte, wurde am 17. Januar 1463 im Torgauer Schloss geboren. Friedrich hat Torgau nach Wittenberg lebenslang besondere Aufmerksamkeit gewidmet. Bereits 23-jährig kam er nach frühem Tod seines Vaters 1486 zur Regierung. Ein Jahr zuvor hatte Kurfürst Ernst in die so genannte Leipziger Teilung gewilligt, die Sachsen seine Vorrangstellung als größtes innerdeutsches Territorium kostete und häufigen Streit mit dem Herzogtum Sachsen auslöste. Von nachhaltiger Wirkung waren die Gründung der Universität Wittenberg 1502 als Ersatz für die nun albertinische Universität Leipzig und später die von Friedrich gestaltete Schutzfunktion für die aufkeimende reformatorische Bewegung. Reichstreue und Vermittlungsgeschick sicherten ihm reichsweit hohes Ansehen. Sein Verzicht auf die Kaiserwahl 1519 machte den Weg frei für die Wahl Karls V.

Auf eine von ihm getroffene Personalentscheidung will ich etwas näher eingehen, weil sie von durchschlagender Bedeutung für die frühe Entwicklungsmöglichkeit der reformatorischen Bewegung wurde, nämlich die Berufung von Georg Spalatin zum Geheimsekretär! Spalatin, humanistisch und historisch gebildet, hatte ein Rechtsstudium absolviert, ehe ihn
Kurfürst Friedrich der Weise 1508 zum Lehrer des Kurprinzen Johann Friedrich und später zum Betreuer weiterer Verwandter bestellte und schließlich 1516 zu seinem Sekretär berief. 1517 schon übertrug ihm der Kurfürst die Kirchen- und Universitätsangelegenheiten. Er wurde der Reisebegleiter des Kurfürsten bei allen Reichstagen ab 1518, sein Vertrauter und Seelsorger, bereits 1518 durch Papst Leo X. mit besonderen Beichtvollmachten ausgestattet. Während einiger Jahre in Wittenberg hatte er eine persönliche Verbindung zu Luther aufgebaut, die ihn später zum einflussreichen Vermittler zwischen diesem und dem Kurfürsten werden ließ. 1522 wurde er Hofkaplan und Hofprediger, woraus sich durchaus eine Zuneigung des kursächsischen Hofes zur lutherischen Predigt ableiten lässt. Außerordentliche Leistungen liegen bei Universitätsreform und Neuordnung der Kirche, die er bereits frühzeitig als Aufgabe der Landesherrschaft ansah. Von historischer Dimension bleibt, dass Spalatin den Kurfürsten dafür gewann, Luther gegen Rom und Kaiser Schutz zu gewähren und umgekehrt Luther in einigen Fällen bewog, bei seiner Polemik auf den Kurfürsten Rücksicht zu nehmen. 1522 hatte Spalatin dem Kurfürsten eine Reihe von Vorschlägen für eine Devise unterbreitet, welche dieser auf eine Medaille setzen wollte, die er als Geschenk für seine Aufenthalte beim Reichsregiment plante. Friedrich der Weise entschied sich für „Verbum Domini manet in aeternum“ (Jes. 40,8; Ps. 119,89). Spalatin korrespondierte mit Melanchthon sogar wegen der Schreibweise „in aeternum“. Luther wünschte im August 1522 die fertige Medaille zu sehen und erhielt nahezu postwendend ein Exemplar als Geschenk des Kurfürsten. In ihrer eingeführten Abkürzung in Versalien wurde die Devise verpflich-
1 Karlheinz Blaschke: Torgau. In: Deutscher Städteatlas, Lieferung II. Nr. 14. Dortmund 1979, Sp. 1-4, hier Sp. 3.

Martin Luther auf der Kanzel darunter die ernestinischen Kurfürsten von Sachsen. Ausschnitt aus dem Croy-Teppich in Greifswald, gefertigt 1554 bis 1556 in Stettin. Von links nach rechts Friedrich der Weise, Johann der Beständige und Johann Friedrich der Großmütige ๑ Universität Greifswald

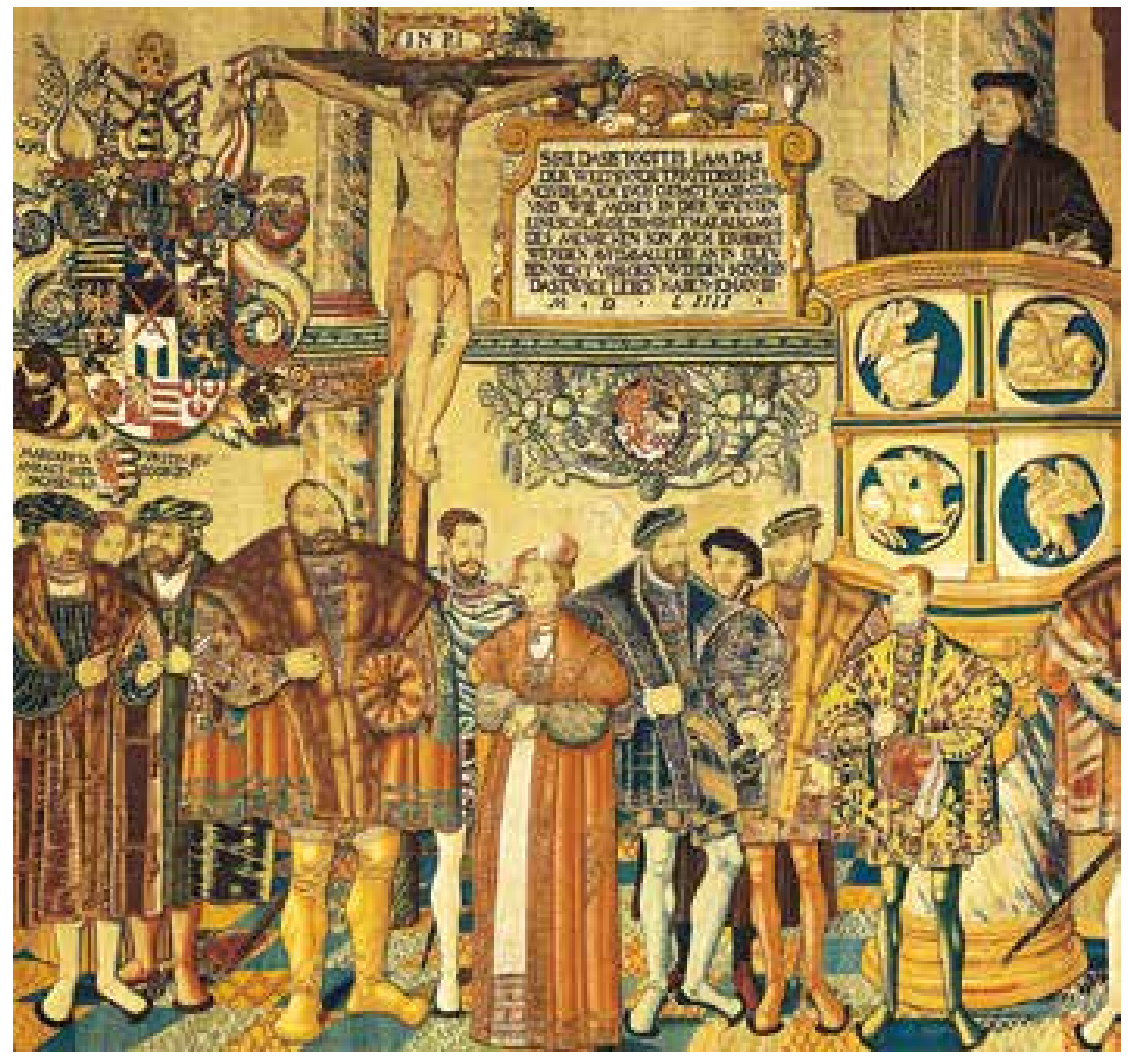


Georg Spalatin, Gemälde von Lucas Cranach, 1509

๑ Museum der Bildenden Künste Leipzig

2 Vgl. Günther Wartenberg: Die reformatorische Veränderung von Kirche und Gesellschaft. Das Werden der Wittenberger Reformation. In: Harald Marx/Cecilie Hollberg (Hrsg.): Glaube und Macht. Sachsen im Europa der Reformationszeit. Aufsätze. Dresden 2004, S. 16-26.

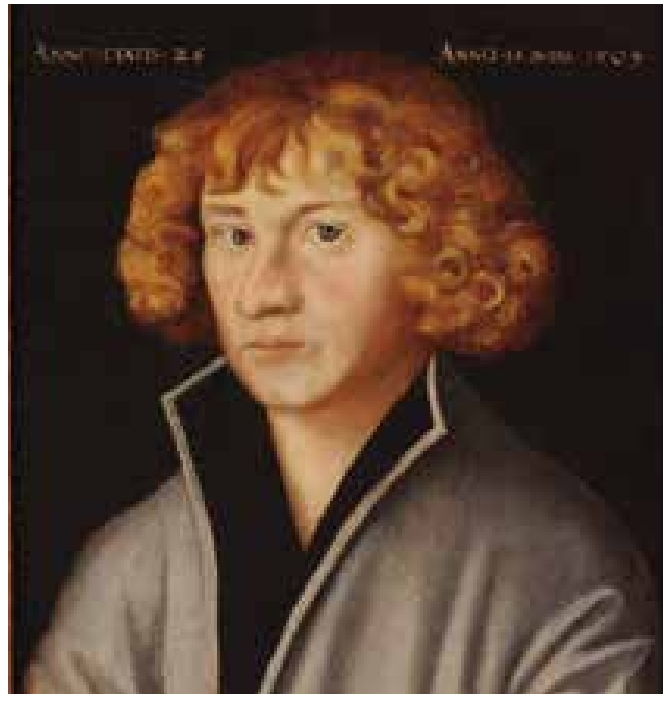

tend für die Hofkleidung bereits im Winter 1522. Beim Reichstag zu Speyer 1526 schlugen die evangelisch gesonnenen Reichsstände die Devise an ihre Herberge. Daraus wurde schließlich die Devise für die Reformation in ganz Europa. Von der Gesamtübersetzung der Bibel durch Martin Luther an stand sie in den Bibelausgaben über dem Druckschutzprivileg des Kurfürsten Johann Friedrich vom Jahre 1534 - und dies über Jahrhunderte. Die Devise steht über den Bronze-Epitaphen der Kurfürsten Friedrich und Johann in der Schlosskirche zu Wittenberg und im Seitenbild des von Lucas Cranach dem Jüngeren gemalten Epitaphs für Kurfürst Johann Friedrich den Großmütigen und seine Gemahlin Sibylle in der Herderkirche in Weimar. Einige Häuser in Torgau tragen die Devise in ihrer markanten Abkürzung V D M I Æ. Spalatins Wohnhaus in Torgau wird noch heute gezeigt.

Die Verwendung der Devise durch den Kurfürsten in den frühen Jahren der Reformation ist ein überzeugendes Merkmal für seine persönliche Haltung. Friedrich der Weise vermied jede institutionelle Förderung der reformatorischen Bewegung, ließ sie jedoch gewähren: in diesem Freiraum konnte sich die neue Theologie ungehindert entwickeln und ausbreiten. ${ }^{2}$

Mit dem Tode Friedrichs des Weisen am 5. Mai 1525 übernahm sein mit ihm gemeinsam regierender Bruder Johann die Regierungsgeschäfte für das gesamte Kurfürstentum allein und verlegte die Residenz dauernd nach Torgau, wo sich seit 1485 die kursächsische Kanzlei befand.

Johann, fünf Jahre jünger als sein Bruder, hatte im Jahre 1500 mit großem Aufwand in Torgau geheiratet, verlor aber nach drei Jahren bei Geburt des Kurprinzen Johann Friedrich seine Gemahlin Herzogin Sophia von Mecklenburg. Aus seiner zweiten Ehe, die zehn Jahre später wiederum in Torgau gefeiert wurde, stammte auch die Tochter Maria, die Luther 1536 in Torgau mit Herzog Philipp I. von Pommern traute.

Nach der Befriedung der Bauernunruhen, die wegen der in Sachsen besseren Situation der bäuerlichen Bevölkerung ruhiger verliefen, schloss Kurfürst Johann zur Absicherung gegenüber der Bedrängnis sei- tens des Kaisers ein Verteidigungsbündnis mit Landgraf Philipp von Hessen, das am 2. Mai 1526 in Torgau ratifiziert wurde. Dem Torgauer Bund traten später weitere Mitglieder bei.

Vor dem Hintergrund der schwierigen außenpolitischen Lage des Reiches und insbesondere der Türkengefahr kam es beim Reichstag zu Speyer 1526 zu einem einstimmigen Reichstagsabschied, mit dem Wormser Edikt gegen Luther und seine Anhänger so zu leben, wie es jeder gegenüber Gott und dem Kaiser zu verantworten hoffe. Die scheinbare Ruhe der Jahre ab 1526 wurde im Kurfürstentum Sachsen für den Aufbau einer evangelischen Landeskirche genutzt. Im gesamten Land sollten Kirchen- und Schulvisitationen stattfinden, für die Spalatin erste Instruktionen für die Visitationsherren entworfen hatte. Sorge war dafür zu tragen, dass Adel und Städte sich nicht am Kirchengut bereicherten und wirtschaftliche Grundlagen geschaffen werden für die Pfarrbesoldung, die Unterhaltung von Schulen und die Wahrnehmung von Sozialaufgaben. Die geistlich wie weltlich gleichsam paritätisch besetzten Visitationskommissionen stellten erschreckende Unkenntnis in Glaubensdingen bei Pfarrern und Gemeindegliedern fest. Die Antwort von Luther kam prompt: Er verfasste einen Großen Katechismus für die Pfarrer und einen Kleinen Katechismus für die Hausväter und zum Auswendiglernen.

Noch im Jahre 1529 wurden die ersten Superintendenten eingesetzt. Torgau erhielt die erste Superintendentur. Die Kirchen- und Schulvisitation im Kurfürstentum Sachsen 1529 gilt als das öffentliche Bekenntnis zur Reformation.

Diese Entwicklung in Kursachsen und eine entsprechende Planung für Hessen als unübersehbare Zeichen für den Fortgang der Reformation veranlassten König Ferdinand I. auf dem zweiten Reichstag zu Speyer 1529, die Aufhebung des Reichstagsabschieds von $1526 \mathrm{zu}$ betreiben. Auf den erwartbaren Beschluss der katholischen Reichstagsmehrheit antworten die evangelisch gesonnenen Reichsstände unter Führung des sächsischen Kurfürsten mit einer feierlichen Protestation: In der Frage des Glaubens könne es einen Mehrheitsbeschluss nicht geben, auch sei ein Reichstagsabschied nicht durch einen einfachen Mehrheitsbeschluss aufhebbar. Seither wird der Ehrenname „Protestanten“ geführt. Da der eher ausgleichend formulierte Beschluss aber ankündigte, beim nächsten Reichstag in Augsburg 1530 schlichten zu wollen, hoffte man protestantischerseits, durch überzeugende Darstellung die Schriftgemäßheit der eigenen Glaubenshaltung dartun zu können.

Kurfürst Johann begehrte von den Wittenberger Theologen eine für den Reichstag geeignete entsprechende Darstellung und außerdem eine Positionierung zu einem möglichen Widerstandsrecht. Bei einer Tagung in der Torgauer Superintendentur brachten Luther, Melanchthon, Justus Jonas und Johannes Bugenhagen die Torgauer Artikel, Grundlage der Augsburgischen Konfession, zur Überreichung an den Kurfürsten zum Abschluss. Melanchthon überarbeitete diese zur Vortragsfassung auf dem Reichstag zu Augsburg. Im Anschluss an die Verlesung durch den kursächsischen Kanzler Beyer ließ der Kaiser durch 


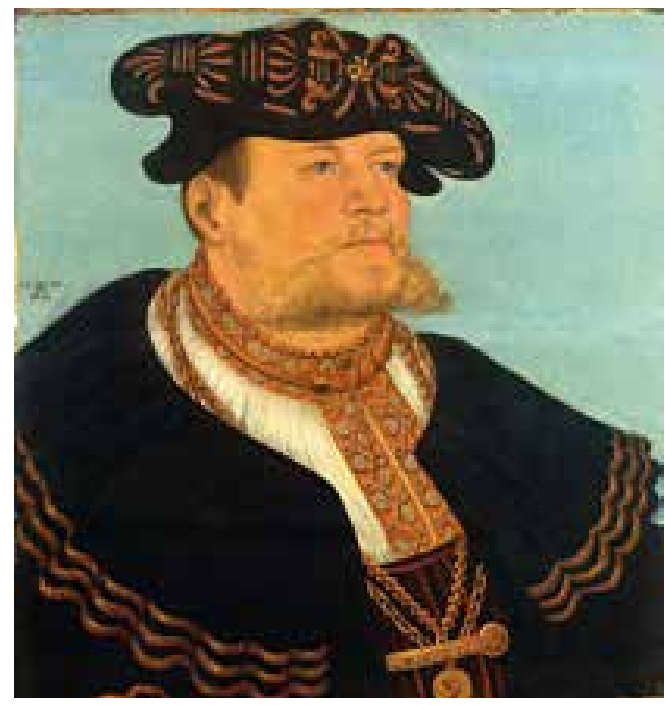

Johann Eck, Luthers theologischen Hauptgegner, die Confutatio (Widerlegung) vortragen und betrachtete die Angelegenheit damit als erledigt. Nach der Entscheidung von Kaiser und Reichstagsmehrheit sollten die Evangelischen binnen eines halben Jahres in die alte Kirche zurückkehren. Mit der Wiederaufnahme der Gewaltandrohung stellte sich für Kurfürst Johann nunmehr die Frage nach dem Widerstandsrecht erneut.

Nachdem im März 1530 in Torgau ein Einvernehmen in dieser Frage nicht hatte bewirkt werden können, wurden die Wittenberger Theologen im Oktober 1530 erneut nach Torgau berufen, um mit den sächsischen Politikern unter Beteiligung von Altkanzler Gregor Brück und Georg Spalatin die rechtlichen Aspekte eines Widerstandsrechts gegenüber dem Kaiser erneut zu beraten.

Die dominierende Persönlichkeit dieser Debatte war zweifelsohne der „Reformationskanzler“ Dr. Gregor Brück, von 1520 bis 1529 kursächsischer Kanzler, danach „Rat von Haus aus“. Seinen Nachfolger, den Wittenberger Professor Dr. Christian Beyer, hatte er zuvor über Jahre eingearbeitet. Beyer trug 1530 beim Augsburger Reichstag die Confessio Augustana vor, weil der Kaiser sich für den Vortrag der deutschen Fassung entschieden hatte. Die lateinische Fassung hätte Brück vorgetragen. Das bekannte Reformatorenbild von Lucas Cranach dem Älteren, um 1532/39, zeigt den Altkanzler unmittelbar neben dem Kurfürsten Johann Friedrich. Von über 1.600 von Brück überlieferten Briefen sind 740 an Johann Friedrich gerichtet. Das reich gestaltete Portal seines Torgauer Hauses mit Darstellung von Kurschwertern und Sachsenraute ist vorzüglich erhalten.

In der Glaubensfrage hatte der Kaiser zur Wiederherstellung der Einheit der Kirche eindeutig mit Gewaltanwendung gedroht. Zur Rechtfertigung eines Widerstandsrechts mussten also die gegen ein solches gerichteten Zweifel ausgeräumt werden.

Luther hatte noch im Frühjahr 1530 darauf verwiesen, dass keine Obrigkeit ohne Gott sei und der Obrigkeit Gehorsam geschuldet werde. Es kam allenfalls der passive Ungehorsam in Betracht, dessen Folgen aber dann zu tragen waren. Kurfürst Johann hatte diese Auffassung Luthers für sich persönlich über- nommen. Von den Wittenberger Theologen vertrat nur Bugenhagen eine andere Auffassung, doch war er im Oktober 1530 im Zusammenhang mit der Neuordnung der kirchlichen Verhältnisse in Lübeck. Wollte man also Kurfürst Johann gewinnen, musste man seinen theologischen Hauptratgeber, nämlich Luther, umstimmen. So hatte Landgraf Philipp von Hessen als aktiver Gegenpart von Luther in dieser Frage das direkte Gespräch mit dem Reformator aufgenommen: Gewaltanwendung in Glaubensfragen ist Unrecht! Gegen Unrechtstaten des Kaisers ist Widerstand zulässig. Die kaiserliche Gewalt ist weniger legitimiert als die der Fürsten; denn der Kaiser kommt durch Wahl der Fürsten ins Amt, diese aber durch Erbfolge. Zwar stimmte Philipp mit Luther in der Überzeugung überein, dass Gott die Seinen nicht im Stich lassen werde, doch sei das Vertrauen auf Gott mit der Nutzung der von Gott bereitgehaltenen Mittel nicht unvereinbar. Genau dieses war die Auffassung der kursächsischen Juristen mit Altkanzler Brück an der Spitze. Mit der verfassungsrechtlichen Argumentation wurde Luther schließlich überzeugt, da das kaiserliche Recht bei offenkundigem Unrecht Gegenwehr zulasse. Als „Torgauer Wende“ ist dieses Ergebnis in die Geschichte eingegangen.

Die so in Torgau erlangte Auffassung wurde in den 1530er Jahren von den Wittenberger Theologen weiter ausgebaut. Die Ableitung des Widerstandsrechts ergab sich zunehmend aus der cura religionis, der Sorge für die rechte Kirche, die zur vornehmsten Aufgabe des Fürsten erklärt wurde. So wurde Widerstandsrecht zur Widerstandspflicht, allerdings immer verstanden als Verteidigung, nicht als Angriff oder als Präventivkrieg. ${ }^{3}$

Als Antwort auf die Kriegsdrohung des Kaisers kam es 1531 zum Schmalkaldischen Bund protestantischer Fürsten und Reichsstädte als Verteidigungsbündnis. Im Nürnberger Anstand von 1532 wurde nochmals Aufschub gewährt, diesmal bis zu einem Konzil. Als Kurfürst Johann am 16. August 1532 starb, hatte er zumindest einen zeitlichen Ausgleich mit dem Kaiser bewirkt.

Wenigstens 61-mal ist Luther in Torgau gewesen. Dass er bei solcher Gelegenheit bei Hofe predigte, war die Regel, mitunter nicht nur einmal. Darüber hinaus ist in vielen Fällen eine weitere Predigt in der Stadtpfarrkirche St. Marien belegt, Luthers „großer Scheune“. Gegen Ende der 1520er Jahre ist die seit dem 14. Jahrhundert nachgewiesene Burgkapelle St. Martin nicht mehr zu gottesdienstlichen Handlungen benutzt worden. Ein Zusammenhang mit der großen Kirchenvisitation und der 1529 offiziell durchgeführten Reformation in Kursachsen wäre einleuchtend. Für 1531 sind Tischlerarbeiten für einen Altar bekundet, der in der Hofstube aufgestellt wurde, also in einem nie für gottesdienstliche Zwecke bestimmten Raum. So war während einiger wichtiger Jahre des reformatorischen Umbruchs in Kursachsen die Obere Hofstube Raum für den Gottesdienst des bewusst evangelischen kursächsischen Hofes. Die an sich original erhaltene gotische Halle ist seit dem 19. Jahrhundert in ihrem nördlichen Drittel um ein Treppenhaus verkleinert, aber mit ihrem schönen Kreuzgratgewölbe immer noch von
Gregor Brück, Gemälde von Lucas Cranach, 1533

( ) Germanisches Nationalmuseum Nürnberg
3 Vgl. Eike Wolgast: Die Torgauer Wende von 1530 zum protestantischen Widerstandsrechtim 16.Jahrhundert. In: Martin Brecht/ Hansjochen Hancke (Hrsg.): Torgau - Stadt der Reformation. o.O. 1996, S. 70-86. 


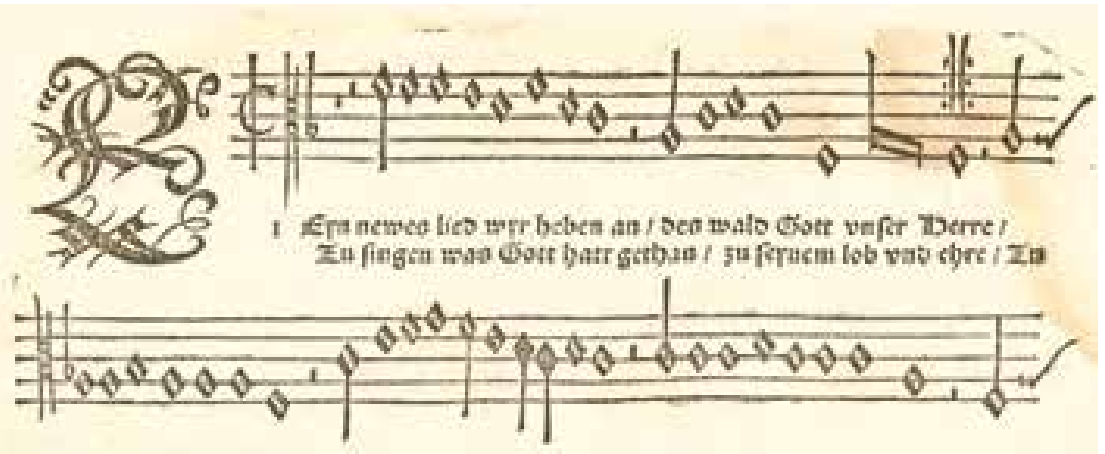

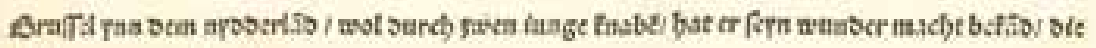

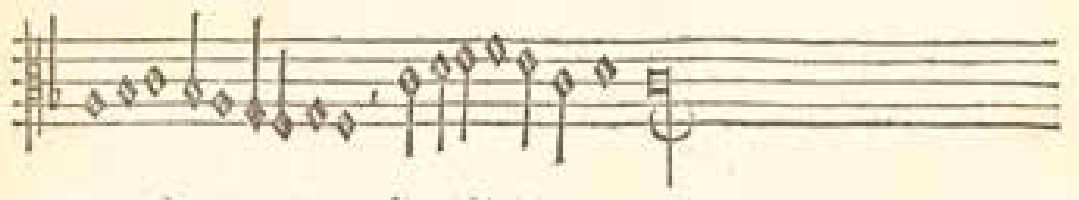

er mitfernen ga beal Go reidstich bat geyret.

Martin Luthers Lied „Eyn newes lied wyr heben an" aus Johann Walters "Geystlich gesangk Buchleyn", 1524 ○ Wikimedia

4 Vgl. Joachim Stalmann: Der erste Musiker der Reformation Johann Walter. In: Armin Schneiderheinze (Hrsg.) Beiheft zu Viue Luthere. Musik der Reformation. Altenburg 1996, S. 2-25, hier S. 8 .

5 Paul Hofmann: Christliches und Torgauisches Jubilate oder Predigt in der Churfürstl. Sächs. Schloß-Capelle zu Torgau. Torgau 1671, zitiert nach dem Wiederabdruck des Vorspanns des Predigtdrucks: Kurze Erzählung der Denkwürdigkeiten der durchlauchtigsten Besitzer des Schlosses zu Torgau, seit den Zeiten Alberts, von Doktor Paul Hofmann, Superintendenten zu Torgau. In: Journal für Sachsen 8 (1792/1793), S. 784-785.

6 Heinz Endermann: Martin Luthers Bibelübersetzung. In: Zu Martin Luther: Biblia. Leipzig 1983, S. 23-34, hier S. $24-25$.

7 Martin Brecht: Martin Luther und Kurfürst Johann Friedrich I. In: Sächsische Heimatblätter 50 (2004), S. 32-41, hier S. 39 eindrucksvoller Ausstrahlung.

In Torgau hatte Luther seinen musikalischen Berater: Johann Walter, den „Urkantor“ der evangelischen Kirche. Mit Auflösung der Hofkapelle Friedrichs des Weisen 1526, in der er Sänger gewesen war, hatte Walter eine Kantorei begründet, in der Mitglieder der Gemeinde und Knaben der Lateinschule sangen. Die musikalische Tradition der Lateinschule ist bereits für das Jahr 1371 nachgewiesen. Johann Walter und die Kantorei haben auch bei Gottesdiensten des Hofes mitgewirkt - wie auch das Engagement Walters 1547 bis zur Sicherstellung des Chorbuchs der Schlosskapelle reicht. Die Mitwirkung der Kantorei bei Luthers Kirchweihe der Schlosskapelle 1544 geriet gar zu einer Art „musikalischen Staatsakts“4; die Huldigungstexte betrafen den Kurfürsten wie auch die beiden Reformatoren Luther und Melanchthon. Paul Hofmann, Superintendent in Torgau, hat in seiner 1671 veröffentlichten Nutzungsgeschichte des Schlosses den von Johann Walter in seine Einweihungsmotette eingefügten lateinischen Lobgesang auf Luther und Melanchthon ins Deutsche übertragen. ${ }^{5}$

Und ein Weiteres ist für Torgau von nachwirkender Bedeutung. Als 1534 die erste Gesamtausgabe von Luthers Bibelübersetzung erschien, kam das kursächsische Druckschutzprivileg mit der Unterschrift Johann Friedrichs aus der Torgauer Kanzlei. Über Jahrhunderte wurde es immer wieder beigedruckt.

Das Torgauer Land inmitten der Mark Meißen lag in einem über Jahrhunderte wirksamen Kolonisationsgebiet, in dem aus oberdeutscher, mitteldeutscher und niederdeutscher Siedlungsherkunft „im Zeitraum weniger Generationen eine Sprachform entstand, die als Verkehrssprache integrierende Kraft erhielt, erfaßte sie doch alle wesentlichen Merkmale des gesamten deutschen Sprachraums ${ }^{\text {"6 }}$ Luther fand hier Anfang des 16. Jahrhunderts Bedingungen vor, die eine rasche Verbreitung seiner Schriften sicherten. Gerade die Bibelübersetzung Luthers nahm somit Einfluss auf die Ausbildung der deutschen Einheitssprache. Seit 1485 war die kursächsische Kanzlei in Torgau untergebracht: Bis etwa 1533/34 in der Alten Kanzlei im Flügel D des Schlosses, da- nach in der neu erbauten Kurfürstlichen Kanzlei vor dem Schloss, dem ersten für diese Behörde errichteten zentralen Verwaltungsbau in Kursachsen.

Als mit dem Tode Kurfürst Johanns sein Sohn Johann Friedrich die Regierung des Kurfürstentums übernahm, fand er bereits eine intakte evangelische Landeskirche vor. Kurz zuvor hatten die von ihm wahrgenommenen Ausgleichsverhandlungen mit dem Kaiser im Nürnberger Anstand eine befristete Sicherheit vor kriegerischen Angriffen aus Glaubensgründen bewirkt. Mit der kursächsischen Politik war er rundum vertraut. Auf sein Amt war er mit Sorgfalt vorbereitet worden, ruhten doch die ernestinischen Erwartungen ganz auf seiner Person.

Kaum ein anderer Reichsfürst war mit der Entwicklung der Reformation so verbunden wie er. Schon als junger Mann nahm er an der schließlich ganz Europa erfassenden und verändernden geistigen Entwicklung und an Martin Luthers persönlichem Schicksal Anteil. Der Reformator war ihm ein „geistlicher Vater“. Das Augsburger Bekenntnis und später die Schmalkaldener Artikel Luthers waren ihm Richtschnur. Außenpolitische Risikospiele wie Bündnispläne mit Frankreich oder England waren ihm suspekt, auch wegen seiner Reichstreue. Dem diplomatischen Ränkespiel der habsburgischen Politik war er allerdings nicht gewachsen. Gegenüber seinem Vetter Moritz von Sachsen versagten ihm Einfühlungsvermögen und Urteilsfähigkeit. Die Rolle Kursachsens im Reich und im Kreis von dessen Territorien war ihm geläufig. „Für sein Amt als erster Fürst unter den reformatorischen Reichsständen war Johann Friedrich geradezu optimal vorbereitet. Er war theologisch gebildet sowie sensibilisiert, und dies auch hinsichtlich der kontroversen Materien." ${ }^{\text {"7 }}$ Er war erklärter Förderer der Universität Wittenberg, den Aufbau der Universitätsbibliothek betrieb er mit Engagement. Er war Schirmherr der Reformation aus Überzeugung - glaubensstark und geradlinig. Sein Schicksal 1547 und seine Haltung in der mehrjährigen kaiserlichen Gefangenschaft machten ihn zum Märtyrer des Protestantismus, seinen Vetter Moritz in den Augen der Zeitgenossen aber zum „Judas von Meißen“.

Die besondere Rolle von Torgau im Reformationszeitalter fand mit der Schlacht bei Mühlberg am 24. April 1547 ein jähes Ende. Herzog Moritz von Sachsen, mit dem Kaiser verbündet, hatte die mögliche Übertragung der sächsischen Kurwürde seines Vetters Johann Friedrich schon vorher abgesichert und regierte danach das um zwei Drittel ernestinischen Gebietes vergrößerte Land von Dresden aus. Torgau wurde Nebenresidenz mit häufigen Aufenthalten der nunmehr albertinischen kurfürstlichen Familie und zahllosen Landtagen in der Stadt. Eine Fürstenzusammenkunft im Mai 1551 auf Schloss Hartenfels diente ihm zur Vorbereitung eines Bündnisses mit König Heinrich II. von Frankreich gegen den Kaiser; im Oktober 1551 trat der französische König dem Bündnis bei. Gegen Abtretung der zum Reich gehörigen Städte Metz, Toul und Verdun flossen die erbetenen französischen Hilfsgelder! Bei dem Feldzug von Moritz gegen den Kaiser im Frühjahr 1552 entging dieser mit Mühe der Gefangennahme. Den Passauer Vertrag vom 2. August 1552 mit König 
Ferdinand I. bestimmten die evangelischen Fürsten. Es war dies die Vorbereitung des Augsburger Religionsfriedens vom 25. September 1555, mit dem die Confessio Augustana rechtlich anerkannt wurde.

Kurfürst Johann Friedrich der Großmütige, wie er schon alsbald nach seinem Tode am 3. März 1554 genannt wurde, war am 30. Juni 1503 im Torgauer Schloss zur Welt gekommen. Er hat, wie er später sagte, Torgau mehr geliebt als alle seine anderen Besitzungen zusammen. Seine glückliche Ehe mit Prinzessin Sibylle von Jülich-Cleve wurde 1526 auf Schloss Burg an der Wupper getraut; am 2. Juni 1527 erfolgte die feierliche Heimholung nach Torgau. Dem Kurfürsten wurden drei Söhne geboren.

Alsbald nach seinem Regierungsantritt hatte Johann Friedrich 1533 mit der großzügigen Neugestaltung der Schlossbebauung begonnen. Der Große Wendelstein des Baumeisters Conrad Krebs gilt als Treppenwunder der Weltarchitektur. Der die Schlosskapelle mit dem Wohnbau des Kurfürsten architektonisch verbindende Schöne Erker von Stefan Hermsdorf zeigt szenische Reliefs und pflanzliche Ornamente in feinster Sandsteinarbeit: Das Bildprogramm bietet eine Art fürstlicher Tugendspiegel - Tapferkeit, Opfermut, Klugheit und Selbstüberwindung allegorisch zum Ausdruck gebracht. Johann Friedrich wurde der Bauherr des ersten großen Schlosses der Frührenaissance in Deutschland. Mit seinem Baumeister Conrad Krebs verbanden ihn nahezu freundschaftliche Beziehungen: der Kurfürst hatte ein Gespür für die Größe dieses Mannes, der weit mehr war als ein herausgehobener Hofbeamter, setzte er doch ein bautheoretisches Konzept um, das den Torgauer Schlossbau in die herausragenden europäischen Schlossbauten der Zeit einreiht.

Zur Vollendung seiner umfangreichen Bautätigkeit hatte Kurfürst Johann Friedrich der Großmütige 1544 einen gottesdienstlichen Raum neu errichten lassen. Ganz in die Fassadengestaltung eines Schlossflügels einbezogen, ist die Kirche des Baumeisters Nickel Gromann äußerlich nur durch das plastisch reiche Portal des Torgauer Bildhauers Simon Schröter als sakraler Bau zu erkennen. Zwischen mittelalterlichen Bergfried und Hofstubenbau mit den kurfürstlichen Wohngemächern eingeschoben, sollte diese Kirche ganz bewusst kein besonderes Haus sein, „als wäre sie besser denn andere Häuser, wo man Gottes Wort predigt“; so sagt es Luther in seiner Kirchweihpredigt. Im Innern zeigt sich eine dreigeschossige überwölbte Halle, umzogen von steinernen Emporen. Unmittelbar gegenüber dem Eingang die Kanzel in der Mitte der streng symmetrisch aufgebauten Längsempore; an der linken Schmalseite mit Ausrichtung nach Nordwesten der Altar und darüber die Orgel; an der rechten Schmalseite übereinander die beiden kurfürstlichen Emporen. Die Schlosskapelle ist mit Ausnahme der Empore des Kurfürsten in ihrem ursprünglichen Zustand erhalten geblieben. ${ }^{8}$

Luthers Torgauer Kirchweihe bedeutete eine eindeutige Abkehr von dem seit Jahrhunderten überkommenen Ritus einer Kirchweihe, ohne Konsekration und ohne Bischof. Predigt, Gebet und Lobgesang standen im Mittelpunkt des Gottesdienstes. Die bauliche Gestaltung der neuen Kirche folgte Luthers Li-

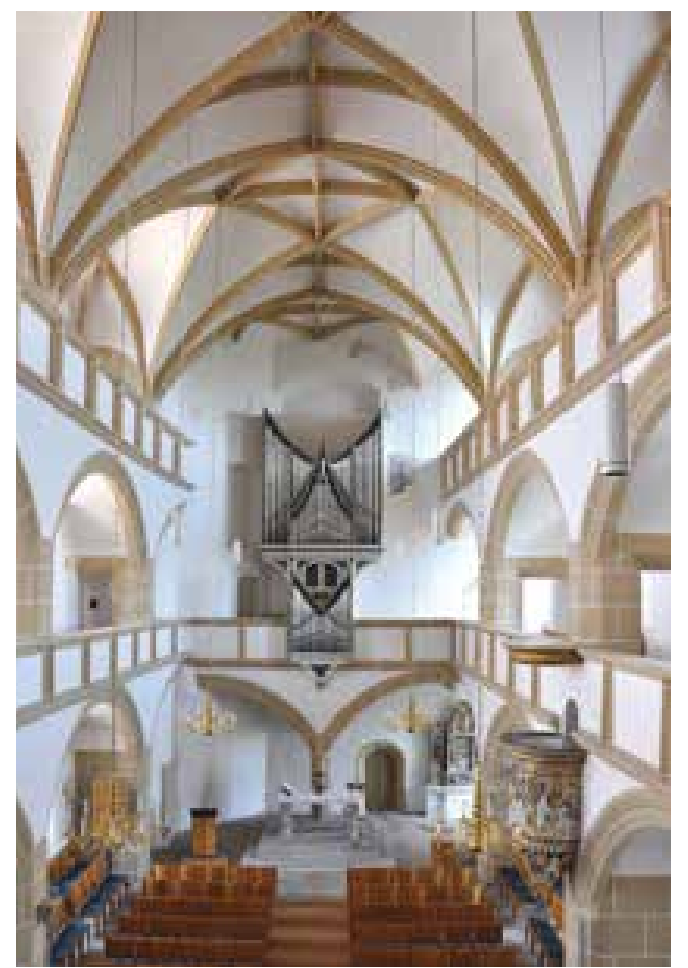

turgievorstellungen, die künstlerische Ausgestaltung war von Cranach mitbestimmt. Es erstaunt nicht, dass die Torgauer Schlosskapelle weltweit am Anfang des protestantischen Kirchenbaus steht und diesen nachhaltig beeinflusst hat. Selten haben reformatorische Bekenntnisse eine derart ausstrahlende kulturelle Umsetzung erfahren.

Unter den Frührenaissancebauten des Schlosses Hartenfels ist nur der Flügel B1 neben seiner äußeren Gestalt auch in seinem Inneren im Wesentlichen erhalten geblieben. Er ermöglicht in besonderer Weise die Erlebbarkeit der historischen Raumsituation und ist somit weit mehr als nur ein Exponat an sich! Dieser Schlossbereich mit dem ersten protestantischen Kirchenneubau, den Kurfürstlichen Gemächern und dem Flaschenturm ist also die einzige wiederherstellbare Nutzungseinheit im Schloss. Die räumliche Verbindung der von Luther geweihten Kapelle mit den persönlichen Räumen des Kurfürstenpaares und dem Flaschenturm ermöglicht an einem wirklichen Brennpunkt die Darstellung historischen Geschehens.

Wittenberg war das geistige Zentrum, Torgau das politische Zentrum der lutherischen Reformation. Genau dieser politische Aspekt ist es, der etwas gefühlsbetont seit dem 19. Jahrhundert mit „Torgau Amme der Reformation“ angesprochen wird. Unter den ernestinischen Kurfürsten war Torgau zur kursächsischen Hauptresidenz und zur Hauptstadt des Kurfürstentums Sachsen geworden. Es waren die Residenzfunktion und das aus dieser resultierende politische Geschehen, das die Solitärstellung von Torgau in der Reformationszeit ausmacht. Sowohl der Rang wie die letztendlich europäische Dimension der von hier beeinflussten reformatorischen Fortentwicklung bestimmen die nachhaltige Bewertung von Torgau in der ersten Hälfte des 16. Jahrhunderts.
Schlosskapelle Torgau, Innenansicht

Foto: Nadja Kühne
8 Hans-Joachim Krause: Die Emporenanlage der Torgauer Schloßkapelle in ihrer ursprünglichen Gestalt und Funktion. In: Bau- und Bildkunst im Spiegel internationaler Forschung. Festschrift zum 80. Geburtstag von Prof. Dr. Edgar Lehmann. Berlin 1989, S. 233-245; Hansjochen Hancke: Spektakulärer Fund zur Architekturgestalt der Torgauer Schloßkapelle. Bedeutende Bildhauerarbeit von Simon Schröter gesichert. In Sächsische Heimatblätter 51 (2005), S. 71-73.

\section{Autor}

Dr. Hansjochen Hancke Torgau 